\title{
Biomarker phenotyping drives clinical management in axillary sentinel node: A retrospective study on women with primary breast cancer in 2002
}

\author{
SERGIO DIOTAIUTI $^{1 *}$, SIMONA DE SUMMA ${ }^{2 *}$, ROSANNA ALTIERI $^{1}$, \\ CATERINA DANTONA $^{1,3}$, STEFANIA TOMMASI ${ }^{2}$, MARIA DI GENNARO $^{4}$, GIUSEPPE RUBINI ${ }^{5}$, \\ MARIA IRENE PASTENA ${ }^{6}$, ANTONELLA ARGENTIERO ${ }^{7}$, FRANCESCO ALFREDO ZITO ${ }^{6}$, \\ NICOLA SILVESTRIS ${ }^{7,8^{* *}}$ and ANGELO VIRGILIO PARADISO ${ }^{4 * *}$
}

\begin{abstract}
${ }^{1}$ Senology Unit; ${ }^{2}$ Molecular Biology and Pharmacogenomics Laboratory, IRCCS Istituto Tumori 'Giovanni Paolo II' of Bari, I-70124 Bari, Italy; ${ }^{3}$ Department of General Surgery, Ospedale Civico di Lugano, 6900 Lugano, Switzerland; ${ }^{4}$ Experimental Oncology Unit, IRCCS Istituto Tumori 'Giovanni Paolo II' of Bari;

${ }^{5}$ Nuclear Medicine Institute, University of Bari ‘Aldo Moro'; ${ }^{6}$ Histopathology Unit, IRCCS Istituto Tumori

'Giovanni Paolo II' of Bari; ${ }^{7}$ Medical Oncology Unit, IRCCS Istituto Tumori 'Giovanni Paolo II' of Bari;

${ }^{8}$ Department of Biomedical Sciences and Human Oncology, University of Bari ‘Aldo Moro', I-70124 Bari, Italy
\end{abstract}

Received October 6, 2019; Accepted April 21, 2020

DOI: $10.3892 / \mathrm{ol} .2020 .11793$

\begin{abstract}
The current study examined if cancer biomarker phenotyping could predict the clinical/pathological status of axillary nodes in women with primary breast cancer. Primary breast cancers from 2002 were analyzed for tumor size, estrogen receptor (ER), progesterone receptor (PgR), Ki-67MIB expression and Her2/neu amplification. Relationships between the clinical and pathological status of the axilla and the biological subtypes classification were analyzed using univariate, multivariate and regression tree analysis. A total of $65 \%$ of women with axillary nodes clinically involved had complete axillary node dissection (ALND) while 705 women with clinically negative axillary underwent sentinel lymph node biopsy (SLNB), $18.5 \%$ of the latter had at least one pathologically SLNB involved node. Multivariate analysis revealed that the Luminal A subtype was significantly associated (OR $\left.0.62 ; \mathrm{P}<10^{-9}\right)$ with clinical negative axilla while HER2pos/not Luminal was associated with clinical positivity (OR $1.71 ; \mathrm{P}<0.01)$. No significant association between biological subtypes and SLNB status was demonstrated. Regression tree analysis revealed that subgroups with significantly different probability of SLNB
\end{abstract}

Correspondence to: Professor Nicola Silvestris, Medical Oncology Unit, IRCCS Istituto Tumori ‘Giovanni Paolo II' of Bari, 65 Viale Orazio Flacco, I-70124 Bari, Italy

E-mail: n.silvestris@oncologico.bari.it

\section{${ }^{* * * *}$ Contributed equally}

Key words: breast cancer, subtyping, sentinel node, axillary node status were separated according to tumor size and $\operatorname{PgR}$ values. In conclusion, the current study demonstrated that biomarker breast cancer phenotyping is significantly associated with clinical status of axillary nodes but not with pathological involvement of nodes at SLNB. Regression tree analysis could represent a valid attempt to individualize some patients subgroups candidate to different surgical axilla approaches.

\section{Introduction}

In the last few years, the therapeutic strategy for primary breast cancer has undergone radical changes. The possibility to characterize breast cancer histological subtypes according to molecular phenotypes as well as the adoption of the technique of axillary sentinel lymph node clearance were among the causes that brought about the innovations (1).

In the early 2000s, Sørlie introduced the concept of biomolecular characterization of primary breast cancer, based on gene expression profile (2). Subsequently, in 2013, the St Gallen International Expert Consensus stated that conventional tumor biomarkers such as hormone receptor status, tumour proliferative activity and Her2/neu gene amplification permitted a surrogate breast cancer subtype classification representative of multigene molecular assays (3). Breast cancers were confirmed to be classified according to different biomarker characteristics in 5 subtypes: Luminal A, Luminal B/HER2 negative, Luminal B/HER2 positive, HER2-positive (not luminal), Basal Like-Triple negative (TNBC). The prognostic-predictive relevance of this subclassification has been largely confirmed (4).

Screening programs and women's increased awareness of breast cancer risk are permitting an even earlier diagnosis of breast cancer, with smaller size primary tumour and lower 
frequency of involved axillary lymph nodes at diagnosis (5). Consequently, to perform a radical surgical clearance of the axilla has been largely debated also the complete axillary node dissection (ALND) is limited by potentially important negative side effects and without a 'per se' clear therapeutic efficacy (6). Currently, sentinel lymph node biopsy (SLNB) has replaced ALND for axillary staging in patients with early-stage breast cancer (7). However, the reported incidence of node metastasis at SLNB is generally low which means that for the most part, women underwent unnecessary invasive axilla surgery (8); furthermore, recent data raises doubts on the role of SLNB itself as clinical determinant, considering that small tumors, after diagnosis of positive sentinel node would not necessarily need further total axillary dissection $(5,9)$.

The purpose of this study is to verify in a large single-center consecutive series of early breast cancer patients if biomarker tumor characterization according to the St. Gallen criteria (3) can play a role in the decision making process regarding the surgical management of axillary lymph node and in particular regarding the use of SLNB.

\section{Materials and methods}

We retrospectively analyzed breast cancer cases consecutively treated with primary surgery at our National Cancer Research Centre between 2008-2014. Clinical and biological information from women enrolled in the study were available: Complete biomarker assay of the primary tumour permitting the breast cancer subtype classification according to St. Gallen 2013 (3); known clinical status of axillary nodes at time of primary surgery; in cases with clinically negative axilla, histological diagnosis of SLNB.

2002/2420 breast cancer patients were enrolled, of whom, $1297(64.8 \%)$ with clinically positive axillary nodes underwent complete ALND; conversely, 705 (35,2\%) women with clinically negative axillary nodes underwent SLNB. The clinical status of axillary nodes was evaluated by the same operator (D.S.). 130/705 cases $(14,6 \%)$ treated with SLNB showed metastasis at least in one of the cleared nodes (median number of cleared nodes $=2.3$ ).

All women had their primary breast cancer analyzed for tumor size ( $\mathrm{mm}$ in max diameter), estrogen receptor (ER) and progesterone receptor $(\mathrm{PgR})$ by ISH, tumour proliferative activity according to Ki-67MIB expression, Her2/neu amplification according to ISH/FISH approach.

The present study was approved by the Institutional Review Board of the National Cancer Research Institute 'Giovanni Paolo II'. Before undergoing routine surgery, all patients signed an informed consent form authorizing the use of the removed biological tissue for research purposes according to ethical standards.

Sentinel Lymph Node preoperative assessment and intraoperative biopsy. In order to detect sentinel lymph nodes, a single tracer was used according to a two-day-protocol. The day before surgery patients were injected subdermally above the breast lesion with $100 \mathrm{MBq}$ Tc-99m-labelled human albumin colloid particles at the Nuclear Medicine Institute of University of Bari. After injection of Tc-99m-labelled human albumin colloid particles the radioactive emission of the tracer in sentinel lymph nodes were identified with a gamma-camera (e.cam, Siemens) and additionally with a hand-held gamma-detector probe and marked on the skin. In additon, pictures including the area of injection and emission of tracer were taken. A hand-held gamma-detector probe was used to identify the sentinel node and guide the surgeon intraoperatively (10). The definition of the sentinel node consisted in any hot node with an ex vivo radioactivity count of ten times or more above the background count with the probe held perpendicular and in direct contact to the node. $\gamma$-probe counts were obtained on axillary dissection specimens, all surrounding nodal basins, and sentinel nodes ex vivo.

Laboratory analyses. After surgical removal the breast tissue was immediately transferred to the Histopathology Unit of our Institute where all analyses were performed by operators with long standing experience in biomarker analysis and in intra-extra laboratory QC programs $(11,12)$. ER, PgR, and Ki-67Mib-1 biomarkers were analysed by immunohistochemical assays according to standards already employed by our team (13). HER2/neu oncogene status was determined according to ASCO/CAP guidelines (14).

In more detail, the expression of $\mathrm{ER}, \mathrm{PgR}$, and of $\mathrm{Ki}-67 \mathrm{Mib}$ cellular proliferation index were tested by IHC. Estrogen and progesterone hormone receptors were tested using monoclonal rabbit anti-human estrogen receptor $\alpha$ (clone SP1; 1:60 dilution; Dako, Glostrup, Denmark) and monoclonal mouse anti-human progesterone receptor (clone PgR 636; 1:100 dilution; Dako) respectively; Ki-67MiB1 was detected using monoclonal mouse anti-human Ki-67 antigen (clone E3 ubiquitin protein ligase 1 (MIB-1); 1:80 dilution; Dako). ER and $\mathrm{PgR}$ receptors were scored as positive when $>1 \%$ of tumor cells were present in the tumor (15). Ki-67MIB1 expression was considered high when staining was present in $>14 \%$ of tumor cells (16).

All samples were analyzed for HER2/neu expression by IHC using the HerceptTest ${ }^{\mathrm{TM}}$ kit (Dako) according to the manufacturers' protocol. HER2 was scored as $0,1^{+}, 2^{+}$or $3^{+}$; for tumors scored $1^{+} / 2^{+}$, FISH for gene amplification was conducted using a dual HER2/Cep17 probe (Path Vysion HER2 DNA Probe kit; Abbott Molecular, Inc., Des Plaines, IL, USA. Gene amplification was reported when HER2/CEP17 ratio was $\geq 2$ or when the mean HER 2 copy number was $\geq 6$.

The Laboratory where the biomarkers assay of breast cancer was performed participated in QC programs for ISH analysis of Hormone Receptors and for FISH analysis of HER/2neu managed by Società Italiana Anatomia Patologica and Citologia Diagnostic.

Statistical analysis. The hormone receptor status, the proliferation activity of Ki-67/MiB-1 expression and the HER2/NEU expression/amplification of the tumor were analyzed by univariate analysis. Frequency of cases in subgroups of patients with different clinical pathological characteristics were compared by $\chi^{2}$-test.

The frequency of clinically positive nodes and histologically positive nodes at SLNB were analyzed separately in the 5 subgroups of tumours by univariate and multivariate logistic regressions. A generalized linear model was fitted 
Table I. Clinical characteristics of the series.

\begin{tabular}{|c|c|c|c|c|c|c|}
\hline \multirow[b]{2}{*}{ Characteristic } & \multicolumn{3}{|c|}{ Axillary clinical status } & \multicolumn{3}{|c|}{ Sentinel lynph node status } \\
\hline & Negative $n=710$ & Positive $n=1294$ & P-value & Negative $n=570$ & Positive $n=130$ & P-value \\
\hline Age (years) & & & $1.003 \times 10^{-9}$ & & & 0.0004 \\
\hline$<59$ & $417(42)^{\mathrm{a}}$ & $574(58)$ & & 318 (76.9) & $95(23.1)$ & \\
\hline$\geq 59$ & $293(28.9)$ & $720(71.1)$ & & $252(87.8)$ & $35(12.2)$ & \\
\hline $\mathrm{T}$ diameter & & & $2.2 \times 10^{-16}$ & & & 0.0001 \\
\hline$\leq 10 \mathrm{~mm}$ & $106(71.1)$ & $43(28.9)$ & & $100(95.2)$ & $5(4.8)$ & \\
\hline$>10$ and $\leq 20 \mathrm{~mm}$ & $340(56.7)$ & $259(43.3)$ & & $281(84.1)$ & $53(15.9)$ & \\
\hline$>20 \mathrm{~mm}$ & $151(22.4)$ & $523(77.6)$ & & $112(75.2)$ & $37(24.8)$ & \\
\hline N/A & $113(19.4)$ & 469 (80.6) & & $77(68.7)$ & $35(31.3)$ & \\
\hline ER status & & & 0.01 & & & 0.49 \\
\hline Negative & 109 (29.9) & $255(70.1)$ & & $91(84.2)$ & $17(15.8)$ & \\
\hline Positive & $601(36.6)$ & $1,039(63.4)$ & & 479 (80.9) & $113(19.1)$ & \\
\hline PgR status & & & 0.02 & & & 0.32 \\
\hline Negative & $187(31.5)$ & 406 (68.5) & & $154(84.1)$ & $29(15.9)$ & \\
\hline Positive & $523(37.1)$ & $888(62.9)$ & & $416(80.5)$ & $101(19.5)$ & \\
\hline HER2/neu amplified & & & $9.72 \times 10^{-6}$ & & & 0.66 \\
\hline Negative & $606(37.8)$ & $996(62.2)$ & & $489(81.7)$ & $109(18.3)$ & \\
\hline Positive & $104(25.8)$ & $298(74.2)$ & & $81(79.4)$ & $21(20.6)$ & \\
\hline Ki-67/Mib1 express & & & $2.1 \times 10^{-11}$ & & & 0.03 \\
\hline Negative & $349(44.4)$ & $437(55.6)$ & & $292(84.6)$ & $53(15.4)$ & \\
\hline Positive & $361(29.6)$ & $857(70.4)$ & & $278(78.3)$ & $77(21.7)$ & \\
\hline
\end{tabular}

a\% in parentheses, $\mathrm{T}$, tumor; ER, estrogen receptor; $\mathrm{PgR}$, progesterone receptor.

by the glm function of R (version 3.2.3) (R Core Team 2015. $\mathrm{R}$ :A language and environment for statistical computing. R Foundation for Statistical Computing, Vienna, Austria. URL https://www.R-project.org/), adjusting for age.

A Regression Tree analysis, which included all the main clinical pathological characteristics such as continuous variables in the model, was performed. The results evidenced the clinico-pathological characteristics and their cut-off values which significantly separated subgroups of cases (nodes) with different percentage of positive SLNB. Results were considered as statistically significant when $\mathrm{P}<0.05$.

\section{Results}

A total of 2002 women participating were involved in this retrospective study, and they were enrolled considering a consecutive series of patients treated for primary breast cancer at our Institute. Median age of the series was 59 years old (range $35-83$ years old) (Table I). $7.4 \%, 30$ and $34 \%$ of women had T1a-b, T1c, and T2 tumors. 81 and $70 \%$ of women resulted positive to $\mathrm{ER}^{+}$and $\mathrm{PgR}$ tumors, respectively. $20 \%$ of the cases resulted HER2/neu positive. A diagnosis of invasive ductal carcinoma was performed in $76,17 \%$; lobular invasive carcinoma represented $7,19 \%$ of cases.

All patients with primary breast cancer included in the analysis were classified in 5 biomarker subtypes according to St Gallen 2013 (3) as reported in Table II. Luminal A and Luminal B/HER negative subtypes resulted to be more frequent (35.4 and 35\%, respectively); conversely, HER2 positive not Luminal were the less frequent $(8.1 \%)$.

Axillary nodes clinical status. Regarding the assessment of axillary lymph node clinical status 1297/2002 (64.8\%) women treated for primary breast cancer at our Institute had clinically positive axillary nodes and were selected to receive complete ALND. This subset of patients was significantly younger, with tumor diameter significantly smaller and biomarker asset expressing more aggressiveness in comparison to women with node negative axilla (Table I).

When biomarker subtyping was considered (Fig. 1), Luminal B/HER2 tumors showed the higher percentage (76.7\%) of women with clinically positive axilla, TNBC the lowest with $63.6 \%$ of the cases. The percentage of clinically positive nodes resulted significantly different between TNBC and HER2/neu positive subgroups $(\mathrm{P}<0.01)$.

To assess the relationships among biomarker subtypes and axillary nodal status independently from other clinico-pathological variables of ascertained clinical relevance, we performed a logistic regression analysis with clinical nodal status such as dependent variable and tumor size, age at diagnosis and subtypes included in the model. The multivariate analysis demonstrated that Luminal A subtype was significantly associated (OR $0.62 ; 95 \%$ CI $0.49-0.78 ; \mathrm{P}<10^{-9}$ ) with a lower probability of clinically positive axillary nodes while 
Table II. Biomarker classification of 2002 primary breast cancers according to Goldirsch et al (3).

\begin{tabular}{lr}
\hline Biomarker subtypes & $\mathrm{N}(\%)$ \\
\hline Luminal A: ER ${ }^{+} \mathrm{PgR}^{+}, \mathrm{MiB}-1$ low, HER2/NEU neg & $709(35.37)$ \\
Luminal B/HER2 negative: $\mathrm{ER}^{+}, \mathrm{PgR}^{+}, \mathrm{MiB}-1$ high, HER2/NEU neg & $701(34.98)$ \\
Luminal B/HER2 positive: $\mathrm{ER}^{+}, \mathrm{PgR}^{+}, \mathrm{MiB}-1$ high/low, HER2/NEU +++ & $239(11.92)$ \\
HER2 positive (not luminal): ER, PgR, HER2/NEU +++ & $163(8.13)$ \\
Triple negative: ER, PgR, HER2/NEU neg. & $192(9.6)$
\end{tabular}

ER, estrogen receptor; PgR, progesterone receptor.

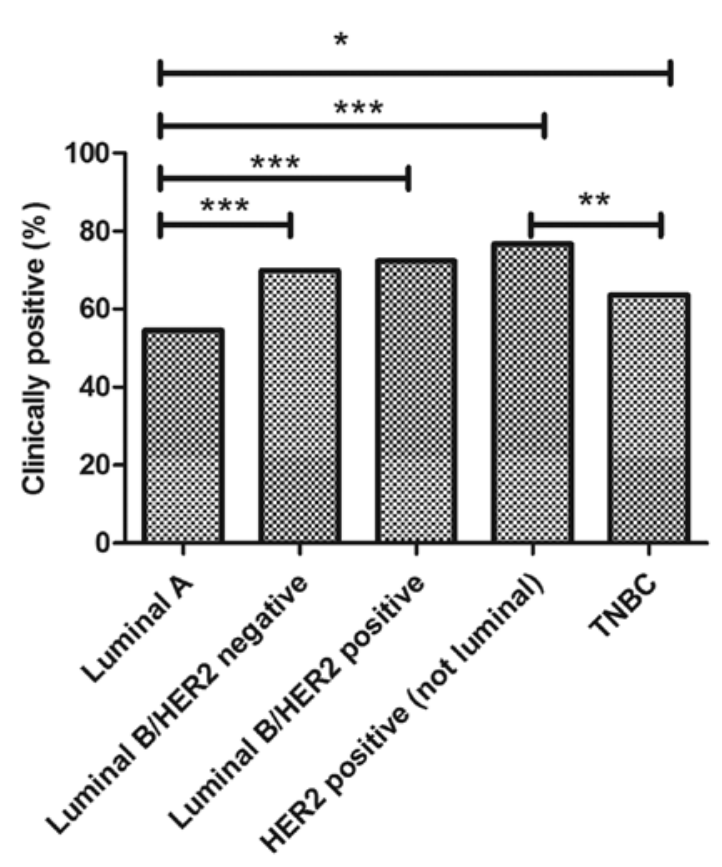

Figure 1. Biomarker subtypes and percentage of patients with clinically positive axilla nodes. Two-proportion $\mathrm{z}$ test assessed whether proportion of clinically positive axilla nodes is significantly different among the distinctive biomarker subtypes. ${ }^{*} \mathrm{P}<0.0001 ;{ }^{* *} \mathrm{P}<0.01 ;{ }^{* * *} \mathrm{P}<0.05$. TNBC, Triple negative breast cancer.

the subgroup of HER2 positive/not Luminal with the highest (OR 1.71; 95\% CI: 1.12-2.65; P<0.012) (Table III).

SLNB status. All women with clinically negative axillary nodes $(n=705)$ underwent SLNB. The detection rate of a sentinel lymph node biopsy assay was $>95 \%$. 130 out of 705 (18.5\%) cases showed metastasis at least in one of the surgically cleared nodes (median number of cleared nodes=2.3). The subgroup of patients having at least one metastatic node at SLNB was older $(\mathrm{P}<0.0004)$ and the node itself was larger in diameter $(\mathrm{P}<0.0001)$ than those without (Table I).

Moreover, the histological SLNB status of nodes was analyzed with respect to biomarker subtypes. Our series of women affected by breast cancer and treated with SLNB $(n=705)$, showed a differently distributed percentage of cases with positive sentinel lymph node in the 5 subgroups (Fig. 2). In particular, the percentage resulted higher in Luminal B/HER2 negative tumors $(23.8 \%)$ and lower in TNBC (14.5\%). The probability of finding metastatic nodes at SLNB resulted significantly

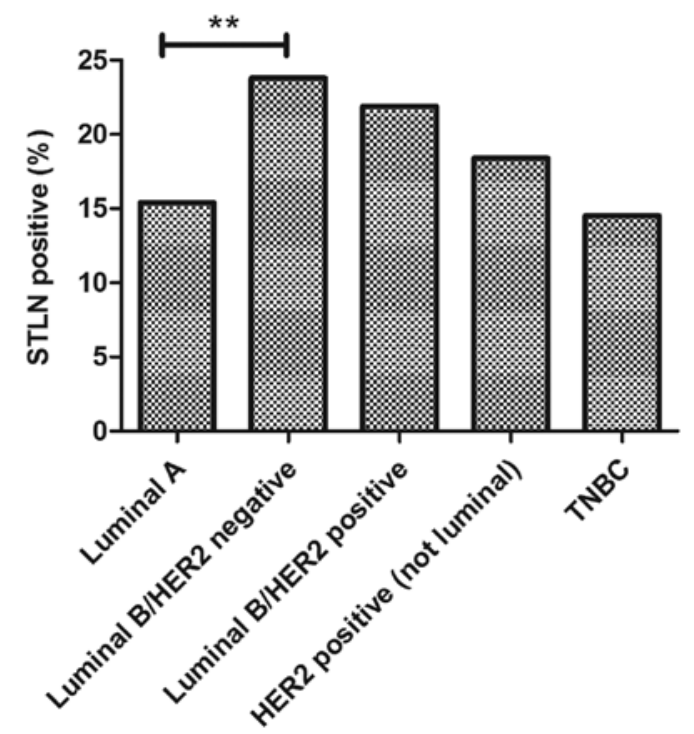

Figure 2. Biomarker subtypes and percentage of patients with positive axillary sentinel lymph node. Two-proportion $\mathrm{z}$ test assessed whether proportion of positive axillary lymph node is significantly different among the distinctive biomarker subtypes. The probability of nodal invasion at sentinel lymph node biopsy deemed significantly different in Luminal A compared to Luminal $\mathrm{B} / \mathrm{HER} 2$ negative subgroups. ${ }^{* *} \mathrm{P}<0.01$. TNBC, Triple negative breast cancer; STLN, sentinel lymph node.

different between Luminal A and Luminal B/HER2 negative subgroups (15.4\% vs. $23.8 \%$, respectively; $\mathrm{P}<0.01)$.

Moreover, the relationships between breast cancer subtypes and SLNB status independently from other clinico-pathological variables of ascertained clinical relevance were investigated by logistic regression analysis considering node positivity as an dependent variable and with tumor size, age at diagnosis, biomarker subtypes included in the model (Table IV). The subtype Luminal B/ HER2 negative showed a strong trend (OR 1.73; 95\% CI: 0.94-3.09; $\mathrm{P}<0.06)$ towards an association with a higher probability for positive SLNB. No additional statistical associations were verified.

Considering the limited power of biomarker subtyping in predicting SLNB status, we employed a regression tree analysis using age, tumor size, ER expression, PgR expression, Ki-67/Mib1 expression as continuous variables, and with HER2/neu status included in the model (Fig. 3).

We highlighted a decisional tree with marker cut-offs indicating 5 subgroups of patients (nodes) significantly different in view of the probability of having positive nodes at SLNB; the 
Table III. Logistic regression with independent variable the clinically positive lymph node status. The five biomarker profiles were analyzed with respect to clinically positive nodal status, when adjusted for tumor size and age (median: 59 years).

\begin{tabular}{lcr}
\hline Biomarker profile & Odds ratio $(95 \%$ CI) & P-value \\
\hline Luminal A & $0.62(0.49 \div 0.78)$ & $<0.001$ \\
Luminal B/HER2 negative & $1.52(1.11 \div 2.10)$ & 0.009 \\
Luminal B/HER2 positive & $1.62(1.11 \div 2.39)$ & 0.011 \\
HER2 ${ }^{+}$(not luminal) & $1.71(1.12 \div 2.65)$ & 0.012 \\
Triple negative & $0.71(0.49 \div 1.04)$ & 0.070 \\
\hline
\end{tabular}

CI, confidence interval.

probability resulted significantly different in tumors with $<38 \mathrm{~mm}$ compared to those with tumor diameter $>38 \mathrm{~mm}(\mathrm{P}<0.001)$. Specifically speaking the probability was the highest $(24 \%)$ in women with tumour size $>38 \mathrm{~mm}$ (representing $1.7 \%$ of cases of the overall series) and the lowest $(2 \%)$ in those with tumor size $<10 \mathrm{~mm}$ (14.9\% of the overall series). Tumors with size $>10$ and $<38 \mathrm{~mm}$ were further split in two nodes according to PgR status (higher or lower than $50 \%$ of positive tumour cells).

\section{Discussion}

The nodal dissemination represents a complex process in which a vicious cycle boosts both the cancer cells and the bystander microenvironment: The recent biological deconvolution of this reciprocal education uncovered peculiar biomarkers to be pivotal in facilitating the malignant cells undisturbed lymph-node invasion. Nodal involvement exemplifies a paradigmatic setting with prognostic and therapeutic consequences in several malignancies (17-20).

Therefore, we were interested in verifying weather biomarker subtyping could impact on the surgical approach to axillary nodes management in breast cancer. To this end, we considered the clinical status of the axilla, which, if positive, implies an ALND. In clinically nodal negative disease, SLNB is routinely performed. However, SLNB low positive rate often prompts unnecessary surgery (21). Thus, there is an urgent need of largely validated markers able to predict SLNB positivity and, confidently, spare invasive approach, also supporting the overall clinical management.

Current literature is lacking in data concerning the search for markers predictive of node status at SLNB. Age confirms to be a predictive marker for axillary node involvement either at axillary dissection or at SLNB (22). In addition, the size of the sentinel node, and the body mass index has been also reported to predict SLNB status $(22,23)$. High differentiation grade $(24)$, mitotic activity $(25,26)$ and lympho-vascular invasion (27) have been also reported.

More recently, the attention has been focused on biomarker subtyping of breast cancer and axillary node involvement demonstrating a strong association with node involvement at ALND (28); conversely, the question is still debated as regards the association with SLNB status with a significant
Table IV. Logistic regression with independent variable Axillary Lymph nodal status. The 5 biomarker profiles were analyzed with respect to clinically positive nodal status, when adjusted for tumor size and age (median: 59 years).

Genomic biomarker profiles Odds ratio (95\% CI) P-value

\begin{tabular}{lll}
\hline Luminal A & $0.82(0.52 \div 1.29)$ & 0.39 \\
Luminal B/HER2 negative & $1.73(0.94 \div 3.09)$ & 0.06 \\
Luminal B/HER2 positive $^{+}$ & $1.63(0.75 \div 3.28)$ & 0.18 \\
HER2 $^{+}$(not luminal) & $0.76(0.25 \div 1.87)$ & 0.58 \\
Triple negative & $0.58(0.24 \div 1.21)$ & 0.18 \\
\hline
\end{tabular}

CI, confidence interval.

relationship reported only for a lower probability of positive nodes in TNBC $(24,29)$. These last studies were performed in limited series of Asian patients and were characterized by a lack of information regarding biological predictors.

We analyzed the relationships between tumour biomarker subtyping and axillary status in a consecutive series of 2002 women with breast cancer, prospectively collected for this purpose. All these assays and histopathological diagnosis were performed in laboratories actively participating in QC programs. The patients cohort characteristics matched clinical and biological determinants, as can be seen in studies already published $(15,16)$.

For breast cancer biomarker subtyping, we used the classification described by Goldirsch et al (3) that has been largely confirmed to be of prognostic-predictive relevance (4). The frequency in our series of patients of various breast cancer subtypes are reported in Table I; when compared to the Yanagawa observation on 363 patients with breast cancer classified according to same criteria (30), we reported a lower percentage of HER2/neu positive tumors (20\% vs. $30 \%$, in our and Janagawa series, respectively).

When considering clinical-pathological factors predicting clinical status of the axillary nodes (Table I), our experience also confirmed that tumour size was appeared to be the most powerful factor predicting clinical involvement of axillary nodes. The same strong relationship was confirmed regarding the age of women at diagnosis showing that during clinical observation earlier onset was associated with significantly higher probability of node involvement. Clinical involvement of axillary nodes was also more significantly frequent in hormone receptor negative and HER2/neu amplified tumors. However, in a multivariate analysis, only the Ki-67-Mib expression resulted significantly predictive for statistics (OR 0.99; 0.98-0.99 95\% CI; P<0.005).

Table I reports data on SLNB status as well. In an univariate analysis, age at diagnosis $(\mathrm{P}<0.0004)$ and tumor size $(\mathrm{P}<0.0001)$ were confirmed significantly associated with presence of involved nodes at SLNB. Regarding the multivariate analysis of biomarkers significantly associated with SLNB status, we already published that only PgR-positive status confirmed to be associated independently from other variables with SLNB status (OR: 0.37; 95\% CI: 0.17-0.72; P<0.005) (31). In this study we further investigated this point with a regression tree analysis. Interestingly, we were able to confirm the 


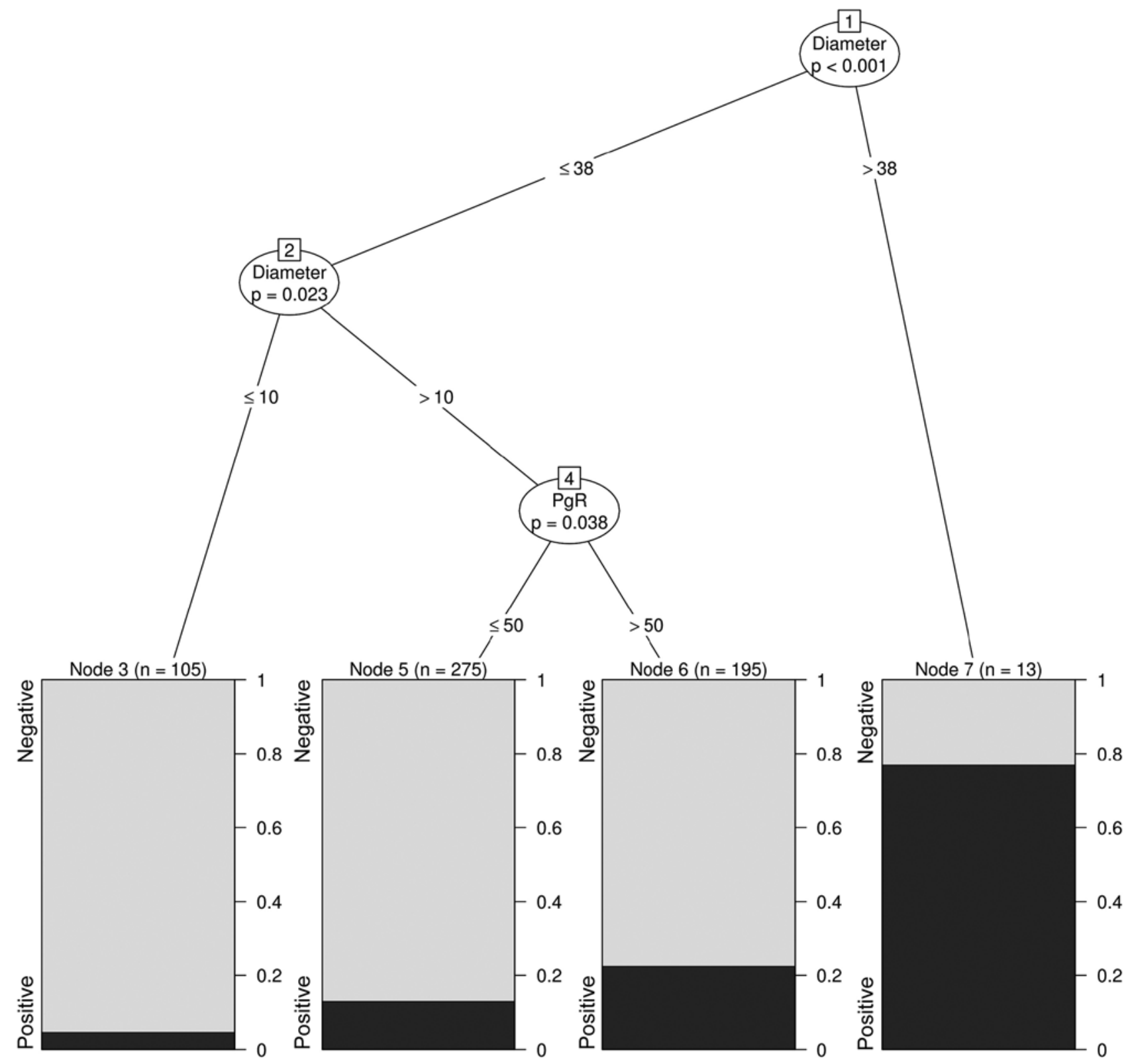

Figure 3. Regression tree for prediction of probability to identify pathological positive axillary nodes in a series of 705 patients with operable breast cancer. Variables included in the model as continuous values: Age, cytohistological differentiation grade, tumor diameter (mm), Ki-67\%, ER and PgR tissue expression (\% of positive cells), Her $2 /$ neu. In leaves indicated number of cases and (in parenthesis) $\%$ of BRCA carriers.

clinical relevance of tumor size and of PgR. (Fig. 3). In fact, we were able to identify tumors moving to it a probability of close to $80 \%$ to have involved nodes at SLNB (tumor diameter $>38 \mathrm{~mm}$ ), to less than $3 \%$ (tumors diameter $<10 \mathrm{~mm}$ ); moreover, in tumors with a diameter comprised between 10 and $38 \mathrm{~mm}, \mathrm{PgR}$ is able to split in two different subgroups. This is the only model demonstrating how it could be possible to manage the SLNB assay according to a combination of tumor size and PgR status data. Interestingly, studies published while our data were being concluded $(24,28,29)$ uncovered the clinico-pathological characteristics to be relevant in the management of lymph node metastasis in breast cancer. To our knowledge, our study substantially extends these findings by providing deeper insight into the largest, monoinstitutional, and consecutive cohort of Caucasian patients. Furthermore, we also employed stringent st Gallen criteria for subtype categorization. These criteria are of paramount importance in describing mammary carcinoma characteristics.

Collectively, we uncovered the age at diagnosis and the tumor size to strong and unequivocal predictors of clinically evident axillary node involvement in our 2002 Caucasian patients cohort. Additionally, tumor biomarkers subtyiping according to Goldirsch classification (3) allowed us to identify Luminal A tumors with a significantly lower probability of clinically evident nodes (OR 0.62).

Conversely, in earlier phases of nodal invasion, characterized by clinically negative axilla, requiring SLNB, tumor size represents a stronger predictor of nodal metastasis. Moreover, we found a lack of clinical utility of additional biomarkers in this scenario. Remarkably, by a regression tree analysis we could dissect subgroups of tumors with different SLNB nodal involvement potential. This approach pinpoints biomarker 
cut-offs for tumour size and PgR status by stratifying four groups of women with variable likelihood of SLNB node involvement ranging from 75 to $3 \%$. This approach hold great clinical promise, prompting statistically powered studies aiming to SLNB optimization.

\section{Acknowledgements}

Not applicable.

\section{Funding}

The current study was supported by Ministry of Health of Italian Government, Funds RC 2017 (grant no. 191/2017) and by the Apulian Regional Project 'Medicina di Precisione' (grant no. 929/2018).

\section{Availability of data and materials}

The datasets used and/or analyzed during the current study are available from the last author on reasonable request.

\section{Authors contributions}

SD and AVP designed the study; RA, CD and MDG collected data and organized the db analysis; MIP and FAZ provided all pathological samples, and analyzed biological data; GR provided all information concerning SLNB; SDS and ST conducted statistical analyses; AVP wrote the manuscript. AA and NS analyzed and interpreted data, revised and edited the work. All authors read and approved the final manuscript.

\section{Ethics approval and consent to participate}

The retrospective study was approved by IAB of the Institute. Before undergoing routine surgery, all patients signed an informed consent form authorizing the use of the removed biological tissue and personal data for research purposes according to ethical standards.

\section{Patient consent for publication}

Not applicable.

\section{Competing interests}

The authors declare that they have no competing interests.

\section{References}

1. Moo TA, Sanford R, Dang C and Morrow M: Overview of breast cancer therapy. PET Clin 13: 339-354, 2018.

2. Sørlie T, Perou CM, Tibshirani R, Aas T, Geisler S, Johnsen H, Hastie T, Eisen MB, van de Rijn M, Jeffrey SS, et al: Gene expression patterns of breast carcinomas distinguish tumor subclasses with clinical implications. Proc Natl Acad Sci USA 98: 10869-10874, 2001

3. Goldhirsch A, Winer EP, Coates AS, Gelber RD, Piccart-Gebhart M, Thürlimann B and Senn HJ; Panel members: Personalizing the treatment of women with early breast cancer: Highlights of the St Gallen International expert consensus on the primary therapy of early breast cancer 2013. Ann Oncol 24: 2206-2223, 2013.
4. Vasconcelos I, Hussainzada A, Berger S, Fietze E, Linke J, Siedentopf F and Schoenegg W: The St. Gallen surrogate classification for breast cancer subtypes successfully predicts tumor presenting features, nodal involvement, recurrence patterns and disease free survival. Breast 29: 181-185, 2016.

5. Jatoi I, Benson JR and Toi M: De-escalation of axillary surgery in early breast cancer. Lancet Oncol 17: e430-e441, 2016.

6. Veronesi U, Viale G, Paganelli G, Zurrida S, Luini A, Galimberti V, Veronesi P, Intra M, Maisonneuve $\mathrm{P}$, Zucca F, et al: Sentinel lymph node biopsy in breast cancer: Ten-year results of a randomized controlled study. Ann Surg 251: 595-600, 2010.

7. Lyman GH, Somerfield MR and Giuliano AE: Sentinel lymph node biopsy for patients with early-stage breast cancer: 2016 American Society of clinical oncology clinical practice guideline update summary. J Oncol Pract 13: 196-198, 2017.

8. Yan M, Abdi MA and Falkson C: Axillary management in breast cancer patients: A comprehensive review of the key trials. Clin Breast Cancer 18: e1251-e1259, 2018.

9. Gentilini $\mathrm{O}$ and Veronesi U: Abandoning sentinel lymph node biopsy in early breast cancer? A new trial in progress at the European Institute of Oncology of Milan (SOUND: Sentinel node vs. observation after axillary UltraSouND). Breast 21: 678-681, 2012

10. Veronesi U, Paganelli G, Viale G, Luini A, Zurrida S, Galimberti V, Intra M, Veronesi P, Maisonneuve P, Gatti G, et al: Sentinel-lymph-node biopsy as a staging procedure in breast cancer: Update of a randomised controlled study. Lancet Oncol 7: 983-990, 2006

11. Paradiso A, Miller K, Marubini E, Pizzamiglio S and Verderio P: The need for a quality control of the whole process of immunohistochemistry human epidermalgrowth factor receptor $2 /$ neu determination: A United Kingdom National External Quality Assessment Service/Italian Network for quality assessment of tumor biomarkers pilot experience. J Clin Oncol 25: e27-e28, 2007.

12. Paradiso A, Volpe S, Iacobacci A, Marubini E, Verderio P,Costa A, Daidone MG, Marchetti A, Mottolese M, Amadori D, et al; Italian network for quality assessment of tumor biomarkers: Quality control for biomarker determination in oncology: The experience of the Italian network for quality assessment of tumor biomarkers (INQAT). Int J Biol Markers 17: 201-214, 2002.

13. Paradiso A, Scarpi E, Malfettone A, Addati T, Giotta F, Simone G, Amadori D and Mangia A: Nuclear NHERF1 expression as a prognostic marker in breast cancer. Cell Death Dis 4: e904, 2013.

14. Lambein K, Van Bockstal M, Denys H and Libbrecht L: 2013 update of the American society of clinical oncology/college of American pathologists guideline for human epidermal growth factor receptor 2 testing: Impact on immunohistochemistry-negative breast cancers. J Clin Oncol 32: 1856-1857, 2014.

15. Hammond MH, Hayes DF, Dowsett M, Allred DC, Hagerty KL, Badve S, Fitzgibbons PL, Francis G, Goldstein NS, Hayes M, et al: American society of clinical oncology/college of American pathologists guideline recommendations for immunohistochemical testing of estrogen and progesterone receptors in breast cancer. J Clin Oncol 28: 2784-2795, 2010.

16. Medri L, Volpi A, Nanni O, Vecci AM, Mangia A, Schittulli F, Padovani F, Giunchi DC, Zito A, Amadori D, et al: Prognostic relevance of mitotic activity in patients with node-negative breast cancer. Mod Pathol 16: 1067-1075, 2003.

17. Stacker SA, Williams SP, Karnezis T, Shayan R, Fox SB and Achen MG: Lymphangiogenesis and lymphatic vessel remodelling in cancer. Nat Rev Cancer 14: 159-172, 2014.

18. Farnsworth RH, Achen MG and Stacker SA: The evolving role of lymphatics in cancer metastasis. Curr Opin Immunol 53: 64-73, 2018.

19. Argentiero A, De Summa S, Di Fonte R, Iacobazzi RM, Porcelli L, Da Vià M, Brunetti O, Azzariti A, Silvestris N and Solimando AG: Gene expression comparison between the lymph node-positive and -negative reveals a peculiar immune microenvironment signature and a theranostic role for WNT targeting in pancreatic ductal adenocarcinoma: A pilot study. Cancers (Basel) 11: 942, 2019.

20. Tjan-Heijnen V and Viale G: The lymph node and the metastasis. N Engl J Med 378: 2045-2046, 2018.

21. Galimberti V, Cole BF, Zurrida S, Viale G, Luini A, Veronesi P, Baratella P, Chifu C, Sargenti M, Intra M, et al: IBCSG 23-01 randomised controlled trial comparing axillary dissection versus no axillary dissection in patients with sentinel-node micrometastases. Lancet Oncol 14: 297-305, 2013. 
22. Sato K, Tamaki K, Shigekawa T, Tsuda H, Kosuda S, Kusano S Hiraide $\mathrm{H}$ and Mochizuki H: Clinicopathologic and technical factors associated with the uptake of radiocolloid by sentinel nodes in patients with breast cancer. Surg Today 33: 403-407, 2003.

23. Schwartz ASK, Leo C, Rufibach K, Varga Z, Fink D and Gabriel N: Does increased tumor burden of sentinel nodes in breast cancer affect detection procedure? Eur J Surg Oncol 39: 266-272, 2013

24. Ding J, Jiang L and Wu W: Predictive Value of clinicopathological characteristics for sentinel lymph Node Metastasis in early breast cancer. Med Sci Monit 23: 4102-4108, 2017.

25. Thangarajah F, Malter W, Hamacher S, Schmidt M, Krämer S, Mallmann P and Kirn V: Predictors of sentinel lymph node metastases in breast cancer-radioactivity and Ki-67. Breast 30: 87-91, 2016.

26. Ozemir IA, Orhun K, Eren T, Baysal H, Sagiroglu J, Leblebici M, Ceyran AB and Alimoglu O: Factors affecting sentinel lymph node metastasis in Turkish breast cancer patients: Predictive value of Ki-67 and the size of lymph node. Bratisl Lek Listy 117: 436-441, 2016.
27. Malter W, Hellmich M, Badian M, Kirn V, Mallmann P and Krämer S: Factors predictive of sentinel lymph node involvement in primary breast cancer. Anticancer Res 38: 3657-3662, 2018.

28. He ZY, Wu SG, Yang Q, Sun JY, Li FY, Lin Q and Lin HX: Breas cancer subtype is associated with axillary lymph node metastasis: A retrospective cohort study. Medicine (Baltimore) 94: e2213, 2015.

29. Mao F, Yao R, Peng L, Zhao JL, Liang ZY and Sun Q: Predictive clinicopathological characteristics affecting sentinel lymph node metastasis in early breast cancer patients. Transl Cancer Res 6: 968-975, 2017

30. Yanagawa M, Ikemot K, Kawauchi S, Furuya T, Yamamoto $S$, Oka M, Oga A, Nagashima Y and Sasaki K: Luminal A and luminal B (HER2 negative) subtypes of breast cancer consist of a mixture of tumors with different genotype. BMC Res Notes 5: 376, 2012.

31. Simone G, Diotaiuti S, Digennaro M, Sambiasi D, De Summa S, Tommasi S, Altieri R, Mangia A, Dantona C and Paradiso A: Comment on 'Renewed interest in the progesterone receptor in breast cancer'. Br J Cancer 117: e1, 2017. 